\title{
LITERATURE REVIEW on WEB PORTAL for ENGINEERING ADMISSION PROCESS
}

\author{
Akshay Mantri ${ }^{1}$, Naina Rohra ${ }^{2}$, Naman Verma ${ }^{3}$, Nikita Gaikwad ${ }^{4}$, Prof. P.V. Kasture ${ }^{5}$ \\ BE Student, Department of Computer Engineering, Sinhgad Academy of Engineering, Pune, India ${ }^{1,2,3,4}$ \\ Professor, Department of computer engineering, Sinhgad Academy of Engineering ${ }^{5}$
}

\begin{abstract}
Education is indispensible for human. Without education one cannot survive in this world. Every year many student land up in wrong colleges because of lack of information. If a system be devised which can provide them proper college information then a number of students taking admission in wrong colleges can be minimize. This system will also provide prediction for the next or future year cutoff list.
\end{abstract}

Keywords: Engineering Admission Process, Ant Algorithm, Prediction Method, Email Sending to massive Destination

\section{INTRODUCTION}

The college admissions environment has changed significantly during the past 20 years. Amid this new landscape, there is growing concern that individual institutional actions, activities of parents, schools, students and other actors in what we refer to as the admission process, may not be serving the values and purposes traditionally associated with higher education. The college admission process has been studied extensively from marketing prospective - how student choose the colleges to which they apply and eventually enroll. Some attention has been given to the psychological aspects of the process - especially the accompanying stress and confusion experienced during the decision making process. However, we now need to better understand the impact of the admission process, especially selective admission, on student learning.

The portal also provides automatic college list generation with the help of preference set. In this we will be providing students who want to take admission through cap rounds, a cap round list according to their preferences of the hostel, mess, placements criteria, marks etc. In email sending module we will send email to students who have registered, advertisement of mess hostel, coaching classes, and we will also send mail for verification of accounts. Cap round list generated can be converted to pdf, excel, text format, and print out can also be taken out. It provides the way to find the nearest hotels, hostels, atm, bank, etc to a particular college. The application will also provide network of all the colleges coming under Pune University autonomous colleges. It will provide timetable for pmt buses, approx auto fair. This paper discuss about the literature review on various algorithms which will be used this application.

\section{LITERATURE REVIEW}

\section{A. Ant Algorithm For Discrete Optimization [1]}

Ant algorithms were first proposed by Dorigo and colleagues as a multi-agent approach to difficult combinatorial optimization problems like the traveling salesman problem (TSP) and the quadratic assignment problem (QAP). Ant algorithms were inspired by the observation of real ant colonies.

The first application of an ant colony optimization algorithm was done using the traveling salesman problem (TSP) as atest.

ACO algorithms

Procedure ACO_Meta_heuristic()

While(termination_criterion_not_satisfied)

\section{Schedule_activities}

Ants_generation_and_activity();

Pheromone_evapration();

Daemon_actions(); \{optional \}

End schedule_activities

End while

End procedure

Procedure ants_generation_and_activity()

While (available_resources)

Schedule_the_creation_of_a_new_ant();

New_active_ant();

\section{End while}

\section{End procedure}

Procedure new_active_ant() \{ ant lifecycle $\}$

Initiate_ant();

M=update_ant_memory(); 
While(current_state!=target_state)

A=read_local_ant_routing_table();

$\mathrm{P}=$ complete_transcation_probabilities(A,M,problem_const raints);

Next_state=apply_ant_decision_policy $(\mathrm{P}$, problem_constra ints);

Move_to_next_state(next_state);

If(online_stepbystep_pheromone_update)

Deposit_pheromone_on_the_visited_arc();

Update_ant-routing_table();

\section{End if}

M=update_internal_state();

\section{End while}

If(online_delayed_pheromone_update)

Evaluate_solution();

Deposit_pheromone_on_all_visited_arcs()

Update_ant-routing_table();

\section{End if}

$\operatorname{Die}()$

\section{End procedure}

\section{B. Rainfall Prediction Using Data Mining [2]}

Data mining is the process of extracting or mining knowledge from large amount of data. In the other words, data mining is the efficient discovery of valuable, nonobvious information from a large collection of data. The goal of data mining is to extract information and convert them into useful knowledge for future information.

\section{Naïve Bayes}

Bayesian classification is a kind of the statistical. It is an algorithm based on the probability. Bayesian algorithm theory is rediscovered and perfected by Laplace, the basic idea is using of the known prior probability and conditional probability density parameter, based on Bayes theorem to calculate the corresponding posterior probability and then obtained the posterior probability to infer and make decisions. Naïve Bayes is a classification model base on the famous Bayes theorem. Naïve Bayes is a tree Bayesian network which contains a root node, a plurality of leaf nodes. In which the leaf node is an attribute variable. It descripts the properties of the object to be classified. The root mode is a class variable that describes the object's categories. The Naïve Bayes classifier is a simple probabilistic classifier based on applying Bayes' theorem with strong (naive) independence assumptions. A more descriptive term for the underlying probability model would be "independent feature model". A naïve Bayes classifier assumes that the presence or absence of a particular feature is unrelated to the presence or absence of any other feature, given the class variable. For some types of probability models, naïve Bayes classifiers can be trained very efficiently in a supervised learning setting. In many practical applications, parameter estimation for naïve Bayes model uses the method of maximum likelihood; in other words, one can work with the naïve Bayes model without accepting Bayesian probability or using any Bayesian methods. Despite their naïve design and apparently oversimplified assumptions, Naïve Bayes classifiers have worked quite well in many complex real-world situations. Bayes method-also called idiot's Bayes, simple Bayes and independence Bayes. This method is important for several reasons. It is very easy to construct, not needing any complicated iterative parameter estimation schemes. This means it may be readily applied to huge data sets. It is easy to interpret, so users unskilled in classifier technology can understand why it is making the classification it makes. And finally, it often does surprisingly well: it may not Probabilistic approaches to classification typically involve modeling the conditional probability distribution. An advantage of Naïve Bayes is that it only requires a small amount of training data to estimate the parameters (means and variance of the variables) necessary for classification.

\section{An Efficient Cloud Design for Email as a Service with} Massive Time Constrain Messages [3]

One of the most widely used communication way around the globe is electronic mail (email) that is more accessible and reachable throughout the world. It is the most suitable way of quick communication for the users, regardless of their level of technical expertise and it provides an automatic delivery service allowing users, separated by location and time, to exchange electronic messages quickly. When the message is needed to be sent in a legal way (excluding to be taken as spam) to a huge number of destinations, it is classified as Massive Email.

The most important idea behind Cloud Computing is scalability and the key technology that makes that possible is virtualization. When the message is sent to various destinations, it is needed to be delivered according to the requirement of a Service Level Agreement (SLA).

In the paper [3], they proposed an efficient cloud design for email as service with massive time constrain messages. They focused their study in solving the problem of sending massive email where we are taking the advantage of the resources that can be offered by a cloud service provider (CSP). This work, they have calculated the number of virtual machine (VMs) that is needed to accomplish the SLA requirement of the client. Furthermore, they performed a deep study of the contention that can be 
International Journal of Advanced Research in Computer and Communication Engineering Vol. 3, Issue 10, October 2014

generated according to the bottleneck of the structure provided trying to find efficient solution to avoid it.

\section{CONCLUSIONS}

Discussing about the hectic schedule of the Students, we decided to develop a web portal which will manage all information regarding colleges. And now we have decided to develop project named "Engineering Admission Counseling "that will effectively help to student for choosing proper college. This will eventually reduce the maximum time requirement of student and providers. As our portal includes the facilities Automatic mail sending, Route finding, View facilities nearer to College etc.

\section{REFERENCES}

[1] Dorigo M, Caro G and Gambardella L "Ant Algorithms for Discrete Optimization" in Artificial Life,Volume:5,Issue:2,DOI:10.1162/106454699568728,Publicatio n Year: 1999,Page(s):137-172,Cited by: Papers(14),MIT PRESS JOURNALS.

[2] Sangari R.S, Dr M. Balamurugan "A Survey on rainfall prediction Using Data Mining " ,International Journal of Computer Science and Mobile Applications on DOI:2,february-2014,Publication Year:2014,Page(s):84-88,vol.2,ISSN:2321-8363.

[3] Gomez Morales M.A., Aymen Abdullah alsaffar, Eui-Nam Huh "An Efficient Cloud Design for Email as a Service with Massive Time Constrain Messages" in Information Technology Research Centre (ITRC) on vol.:76 no.3,2014, publication Year:2014,Page(s):1 15 ,Pensee journal 